\title{
Compendium of Current Total Ionizing Dose and Displacement Damage Results from NASA Goddard Space Flight Center and NASA Electronic Parts and Packaging Program
}

\author{
Alyson D. Topper, Michael J. Campola, Dakai Chen, Megan C. Casey, Ka-Yen Yau, Donna J. Cochran, Kenneth
} A. LaBel, Raymond L. Ladbury, Timothy K. Mondy, Martha V. O’Bryan, Jonathan A. Pellish, Edward P. Wilcox, Edward J. Wyrwas, and Michael A. Xapsos

\begin{abstract}
Total ionizing dose and displacement damage testing was performed to characterize and determine the suitability of candidate electronics for NASA space utilization. Devices tested include optoelectronics, digital, analog, linear bipolar devices, and hybrid devices.
\end{abstract}

Index Terms- Displacement Damage, Optoelectronics, Proton Damage, Single Event Effects, and Total Ionizing Dose.

\section{INTRODUCTION}

Long term radiation induced failure modes play a significant role in determining space system reliability. Therefore, the effects of total ionizing dose (TID) and displacement damage dose (DDD) need to be evaluated through ground-based testing in order to determine risk to spaceflight applications.

The test results presented here were gathered to establish the sensitivity of candidate spacecraft electronics to TID and/or DDD. Proton-induced degradation, dominant for most NASA missions, is a mix of ionizing (TID) and non-ionizing damage. The non-ionizing damage is commonly referred to as DDD. For similar results on single event effects (SEE), a companion paper has also been submitted to the 2017 IEEE NSREC Radiation Effects Data Workshop entitled: "Compendium of Current Single Event Effects Results from

Manuscript received July 12, 2017. This work was supported in part by the NASA Electronic Part and Packaging Program (NEPP) and NASA Flight Projects.

Alyson D. Topper, Donna J. Cochran, Martha V. O’Bryan, and Edward P. Wilcox are with ASRC Federal Space and Defense, Inc. (AS\&D, Inc.), work performed for NASA Goddard Space Flight Center, Code 561.4, Greenbelt, MD 20771 (USA), phone: 301-286-5489, email: Alyson.d.topper@nasa.gov.

Michael J. Campola, Megan C. Casey, Kenneth A. LaBel, Raymond L. Ladbury, Jonathan A. Pellish, Michael A. Xapsos are with NASA/GSFC, Code 561.4, Greenbelt, MD 20771 (USA), phone: 301-286-5427, email: Michael.j.Campola@nasa.gov.

Dakai Chen was with NASA Goddard Space Flight Center, Code 561, Greenbelt, MD 20771. He is now with Analog Devices Inc., Milpitas, CA 95035 (Tel: 408-432-1900 ext. 2191; Dakai.chen@analog.com).

Ka-Yen Yau was with ASRC Federal Space and Defense, Inc. (AS\&D, Inc.)

Timothy K. Mondy is with NASA/GSFC, Code 562, Greenbelt, MD 20771 (USA), phone: 301-286-4253, email: timothy.mondy@nasa.gov

Edward Wyrwas is with Lentech, Inc., P.O Box 67155, Albuquerque, NM 87193, work performed for NASA Goddard Space Flight Center, Code 561.4, Greenbelt, MD 20771 (USA), phone: 301-286-5213, email: Edward.j.wyrwas@nasa.gov.
NASA Goddard Space Flight Center and NASA Electronic Parts and Packaging Program" by M. O’Bryan, et al. [1]

\section{TEST TECHNIQUES AND SETUP}

\section{A. Test Method}

Unless otherwise noted, all tests were performed at room temperature and with nominal power supply voltages. Based on the application, samples were tested in a biased or unbiased configuration. Functionality and parametric changes were measured either continually during irradiation (in-situ) or after step irradiations (for example: every 10 $\operatorname{krad}(\mathrm{Si})$, or every $1 \times 10^{10}$ protons $/ \mathrm{cm}^{2}$ ).

\section{B. Test Facilities - TID}

TID testing was performed using MIL-STD-883, Test Method 1019.9 [2] unless otherwise noted as research. Dose rates used for testing were between 0.05 and $50 \mathrm{rad}(\mathrm{Si}) / \mathrm{s}$.

\section{Test Facilities - Proton}

Proton damage tests were performed on biased and unbiased devices. Table I lists the proton damage test facilities and energies used on the devices.

\begin{tabular}{|l|c|}
\multicolumn{1}{|c|}{ TABLE I: Proton TEST FACILITIES } \\
\begin{tabular}{|l|c|}
\hline \multicolumn{1}{|c|}{ Facity } & $\begin{array}{c}\text { Proton Energy, } \\
(\mathbf{M e V})\end{array}$ \\
\hline $\begin{array}{l}\text { University of California at Davis (UCD) } \\
\text { Crocker Nuclear Laboratory (CNL) }\end{array}$ & 63 \\
\hline Texas A\&M University Cyclotron (TAMU) & 45 \\
\hline
\end{tabular}
\end{tabular}

\section{TEST RESULTS OVERVIEW}

Abbreviations for principal investigators (PIs) are listed in Table II. Abbreviations and conventions are listed in Table III. Summary of TID and DDD test results are listed in Table IV and VI. Summary of on-going TID test results are listed in Table V. Please note that these test results can depend on operational conditions.

TABLE II: LIST OF PRINCIPAL INVESTIGATORS

\begin{tabular}{|c|c|}
\hline Abbreviation & Principal Investigator (PI) \\
\hline DC & Dakai Chen \\
\hline KY & Ka-Yen Yau \\
\hline MCC & Megan C. Casey \\
\hline MJC & Michael J. Campola \\
\hline
\end{tabular}

To be published in the Institute of Electrical and Electronics Engineers (IEEE) Nuclear and Space Radiation Effects Conference (NSREC), Radiation Effect Data Workshop proceedings, New Orleans, Louisiana, July 17-21, 2017. 
TABLE III: ACRONYMS

\begin{tabular}{|l|l|}
\hline A = Amp & LBNL = Lawrence Berkeley National Laboratory \\
BiCMOS = Bipolar - Complementary Metal Oxide Semiconductor & LDC = Lot Date Code \\
BJT = Bipolar Junction Transistor & LDO = Low Dropout \\
CMOS = Complementary Metal Oxide Semiconductor & LED = Light Emitting Diode \\
COTS = Commercial off-the-shelf & LDR = Low Dose Rate \\
CTR = Current Transfer Ratio & LDR EF = Low Dose Rate Enhancement Factor \\
DDD = Displacement Damage Dose & MeV = Mega Electron Volt \\
DDR = Double-Data-Rate (a type of SDRAM-Synchronous Dynamic & mA = milliamp \\
Random Access Memory) & MOSFET = Metal Oxide Semiconductor Field Effect Transistor \\
DTRA = Defense Threat Reduction Agency & Mrad = mega rad \\
DUT = Device Under Test & n/a = Not Available \\
ELDRS = Enhanced Low Dose Rate Sensitivity & Op-Amp = Operational Amplifier \\
FET = Field Effect Transistor & PI = Principal Investigator \\
GSFC = Goddard Space Flight Center & PMU = Pulse Measurement Unit \\
HDR = High Dose Rate & REAG = Radiation Effects \& Analysis Group \\
$\mathrm{h}_{\mathrm{FE}}=$ Forward Current Gain & RF = Radio Frequency \\
$\mathrm{I}_{\mathrm{b}}=$ Base Current & SEE = Single Event Effects \\
$\mathrm{I}_{\mathrm{c}}=$ Collector Current & SMD = Standard Microcircuit Drawings \\
$\mathrm{I}_{\mathrm{CE}}=$ Collector-Emitter Current & Spec = Specification(s) \\
$\mathrm{I}_{\mathrm{os}}=$ Offset Current & TAMU = Texas A\&M University Cyclotron TAMU) \\
$\mathrm{I}_{\mathrm{off}}=$ Dark Current & TID = Total Ionizing Dose \\
$\mathrm{I}_{\mathrm{OUT}}=$ Output Current & UCD-CNL = University of California at Davis - Crocker Nuclear Laboratory \\
JFET = Junction Field Effect Transistor & \\
\hline
\end{tabular}


TABLE IV: SUMMARY OF TID TEST RESULTS

\begin{tabular}{|c|c|c|c|c|c|c|c|c|c|}
\hline Part Number & Manufacturer & LDC & $\begin{array}{l}\text { Device } \\
\text { Function }\end{array}$ & Technology & PI & Results & $\begin{array}{l}\text { App. } \\
\text { Spec } \\
(\mathbf{Y} / \mathbf{N})\end{array}$ & $\begin{array}{c}\text { Dose rate } \\
(\operatorname{mrad}(\mathrm{Si}) / \mathrm{s})\end{array}$ & $\begin{array}{c}\text { Degradation } \\
\text { Level (krad } \\
\text { (Si)) }\end{array}$ \\
\hline \multicolumn{10}{|c|}{ OPERATIONAL AMPLIFIERS } \\
\hline AD654 & Analog Devices & 0630; (16-036) & $\begin{array}{l}\text { Operational } \\
\text { Amplifier }\end{array}$ & Bipolar & MJC & $\begin{array}{l}\text { All parameters within } \\
\text { specification up to } 40 \mathrm{krad}(\mathrm{Si})\end{array}$ & $\mathrm{Y}$ & 10 & $>40$ \\
\hline PA02 & APEX & $1417 ;(16-033)$ & $\begin{array}{l}\text { Operational } \\
\text { Amplifier }\end{array}$ & Bipolar & DC & $\begin{array}{c}\text { All parameters within } \\
\text { specification up to } 30 \mathrm{krad}(\mathrm{Si})\end{array}$ & $\mathrm{Y}$ & 10 & $>30$ \\
\hline LTC6268-10 & Linear Technology & 1433; (16-040) & $\begin{array}{l}\text { Operational } \\
\text { Amplifier }\end{array}$ & BiCMOS & DC & $\begin{array}{l}\text { Minimal degradation up to } 20 \\
\operatorname{krad}(\mathrm{Si})\end{array}$ & $\mathrm{Y}$ & 10 & $>20$ \\
\hline \multicolumn{10}{|l|}{ TRANSISTORS } \\
\hline 2N2907AUB & Microsemi & n/a; (16-022) & $\begin{array}{c}\text { PNP } \\
\text { Transistor }\end{array}$ & Bipolar & KY & $\begin{array}{l}\text { Gain degradation and failures at } \\
45 \operatorname{krad}(\mathrm{Si})\end{array}$ & $\mathrm{Y}$ & 10 & 45 \\
\hline JANTXV2N2222AUB & Microsemi & 1523: (16-021) & $\begin{array}{c}\text { NPN } \\
\text { Transistor }\end{array}$ & Bipolar & KY & $\begin{array}{c}\text { Gain out of specification at } 55 \\
\operatorname{krad}(\mathrm{Si})\end{array}$ & $\mathrm{Y}$ & 10 & $45<X<55$ \\
\hline JANTXV2N5115 & Solitron & 1449A; (16-039) & JFET & Bipolar & MJC & $\begin{array}{l}\text { Minimal degradation up to } 30 \\
\operatorname{krad(Si)}\end{array}$ & Y & 10 & $>30$ \\
\hline \multicolumn{10}{|l|}{ MEMORY } \\
\hline $\begin{array}{l}\text { MT29F128G08AJAAAWP- } \\
\text { ITZ }\end{array}$ & Micron & 201504; (16-017) & Flash & CMOS & MJC & \begin{tabular}{|c|} 
Large number of block errors at \\
$30 \mathrm{krad}(\mathrm{Si})$, Three devices \\
showed unrecoverable chip \\
select errors at 40 krad(Si)
\end{tabular} & $\mathrm{N}$ & $\begin{array}{l}0.7-10 \\
\operatorname{rad}(\mathrm{Si}) / \mathrm{s}\end{array}$ & 30 \\
\hline $\begin{array}{l}\text { MT29F128G08AJAAAWP- } \\
\text { ITZ }\end{array}$ & Micron & $\begin{array}{c}\text { 201504BYGGFZR.21; } \\
(16-018)\end{array}$ & Flash & CMOS & MJC & \begin{tabular}{|c|} 
Two devices showed \\
unrecoverable chip select errors
\end{tabular} & $\mathrm{N}$ & $\begin{array}{l}0.7-10 \\
\operatorname{rad}(\mathrm{Si}) / \mathrm{s}\end{array}$ & 40 \\
\hline MB85AS4MT & Fujitsu & 1638; (16-041) & $\begin{array}{l}\text { Memory - } \\
\text { Nonvolatile }\end{array}$ & $\begin{array}{l}\text { CMOS \& } \\
\text { ReRAM }\end{array}$ & DC & $\begin{array}{c}\text { No memory corruption } \\
\text { observed. Peripheral circuitry } \\
\text { failure observed }>20 \text { krad(Si). }\end{array}$ & $\mathrm{N}$ & $50 \mathrm{rad}(\mathrm{Si}) / \mathrm{s}$ & $20<X<50$ \\
\hline \multicolumn{10}{|l|}{ MISCELLANEOUS } \\
\hline AD2S80 & Analog Devices & 1452; (15-088) & $\begin{array}{l}\text { Resolver to } \\
\text { Digital } \\
\text { Converter }\end{array}$ & BiCMOS & DC & \begin{tabular}{|c|} 
Biased parts show functional \\
failure between 18 and 30 \\
krad(Si) at high dose rate and 12 \\
to 18 krad(Si) at low dose rate.
\end{tabular} & Y & $\begin{array}{c}50 \mathrm{rad}(\mathrm{Si}) / \mathrm{s} \\
\text { and } 10\end{array}$ & $12<\mathrm{FF}<18$ \\
\hline UC1823A & Texas Instruments & 1345; (15-062) & $\begin{array}{c}\text { Pulse Width } \\
\text { Modulator }\end{array}$ & BiCMOS & DC & $\begin{array}{c}\text { All parameters within } \\
\text { specification up to } 30 \mathrm{krad}(\mathrm{Si})\end{array}$ & $\mathrm{Y}$ & 10 & $>30$ \\
\hline
\end{tabular}




\begin{tabular}{|c|c|c|c|c|c|c|c|c|c|}
\hline Part Number & Manufacturer & LDC & $\begin{array}{c}\text { Device } \\
\text { Function }\end{array}$ & Technology & PI & Results & $\begin{array}{l}\text { App. } \\
\text { Spec } \\
(\mathbf{Y} / \mathbf{N})\end{array}$ & $\begin{array}{c}\text { Dose rate } \\
(\mathrm{mrad}(\mathrm{Si}) / \mathrm{s})\end{array}$ & $\begin{array}{c}\text { Degradation } \\
\text { Level (krad } \\
\text { (Si)) }\end{array}$ \\
\hline SW15-802 & $\begin{array}{c}\text { Southwest Research } \\
\text { Institute }\end{array}$ & 1203, 1233; (16-007) & Optocoupler & Hybrid & MCC & $\begin{array}{l}\text { One unbiased part showed an } \\
\text { increase in dark current at } 75 \\
\text { krad(Si). Parameters increased } \\
\text { with dose for biased parts. }\end{array}$ & $\mathrm{Y}$ & $\begin{array}{c}5-50 \\
\operatorname{rad}(\mathrm{Si}) / \mathrm{s}\end{array}$ & $8<X<75$ \\
\hline AD9364 & Analog Devices & 1401; (15-071) & Transceiver & CMOS & DC & $\begin{array}{c}\text { Parameters within specification. } \\
\text { Transmission power gain } \\
\text { showed minimal degradation as } \\
\text { dose increased }\end{array}$ & $\mathrm{Y}$ & $100 \mathrm{rad}(\mathrm{Si}) / \mathrm{s}$ & $>50$ \\
\hline
\end{tabular}

TABLE VI: SUMMARY OF ELDRS TEST RESULTS

\begin{tabular}{|c|c|c|c|c|c|c|c|c|c|}
\hline Part Number & Manufacturer & LDC & $\begin{array}{c}\text { Device } \\
\text { Function }\end{array}$ & $\begin{array}{c}\text { Technology } \\
\text { /Package }\end{array}$ & PI & Results & $\begin{array}{l}\text { App. } \\
\text { Spec } \\
(\mathrm{Y} / \mathrm{N})\end{array}$ & $\begin{array}{c}\text { Dose rate } \\
(\operatorname{mrad}(\mathrm{Si}) / s)\end{array}$ & $\begin{array}{l}\text { Degradation } \\
\text { Level (krad } \\
\text { (Si)) }\end{array}$ \\
\hline \multicolumn{10}{|c|}{ OPERATIONAL AMPLIFIERS } \\
\hline \multirow[b]{2}{*}{ RH1013MH } & \multirow[b]{2}{*}{ Linear Technology } & \multirow[b]{2}{*}{ 0329A; (A214) } & \multirow[b]{2}{*}{$\begin{array}{l}\text { Operational } \\
\text { Amplifier }\end{array}$} & \multirow{2}{*}{$\begin{array}{l}\text { Bipolar / } \\
\text { TO-5 Metal } \\
\text { Can }\end{array}$} & \multirow[b]{2}{*}{ DC } & \multirow{2}{*}{$\begin{array}{c}\text { Small levels of dose } \\
\text { rate sensitivity in the } \mathrm{I}_{\mathrm{B}} \\
\text { degradation. Parameters } \\
\text { within spec. }\end{array}$} & \multirow[b]{2}{*}{$\mathrm{Y}$} & 1 & $>20$ \\
\hline & & & & & & & & 0.5 & $40<\mathrm{I}_{\mathrm{B}} \leq 60$ \\
\hline \multirow[t]{2}{*}{ RH1013MJ8 } & \multirow[t]{2}{*}{ Linear Technology } & \multirow[t]{2}{*}{ 0305A; (A214) } & \multirow[t]{2}{*}{$\begin{array}{c}\text { Operational } \\
\text { Amplifier }\end{array}$} & \multirow{2}{*}{$\begin{array}{l}\text { Bipolar } \\
\text { /Ceramic } \\
\text { DIP }\end{array}$} & \multirow[t]{2}{*}{ DC } & \multirow{2}{*}{$\begin{array}{l}\text { Small levels of dose } \\
\text { rate sensitivity in the } \mathrm{I}_{\mathrm{B}} \\
\text { degradation. Parameters } \\
\text { within spec. }\end{array}$} & \multirow[t]{2}{*}{$\mathrm{Y}$} & 1 & $>20$ \\
\hline & & & & & & & & 0.5 & $40<\mathrm{I}_{\mathrm{B}} \leq 60$ \\
\hline \multirow[b]{2}{*}{ RH1078MH } & \multirow[b]{2}{*}{ Linear Technology } & \multirow[b]{2}{*}{ 0741A; (A224) } & \multirow[b]{2}{*}{$\begin{array}{c}\text { Operational } \\
\text { Amplifier }\end{array}$} & \multirow[b]{2}{*}{$\begin{array}{l}\text { Bipolar /TO- } \\
5\end{array}$} & \multirow[b]{2}{*}{ DC } & \multirow{2}{*}{$\begin{array}{c}\text { Parameters remain } \\
\text { within post-irradiation } \\
\text { specification. } \\
\text { Completed 11/22/2016. }\end{array}$} & \multirow[b]{2}{*}{$\mathrm{Y}$} & 1 & $>40$ \\
\hline & & & & & & & & 0.5 & $>30$ \\
\hline \multirow[b]{2}{*}{ RH1078W } & \multirow[b]{2}{*}{ Linear Technology } & \multirow[b]{2}{*}{ 0325A; (A224) } & \multirow[b]{2}{*}{$\begin{array}{c}\text { Operational } \\
\text { Amplifier }\end{array}$} & \multirow[b]{2}{*}{$\begin{array}{l}\text { Bipolar } \\
\text { /Flatpack }\end{array}$} & \multirow[b]{2}{*}{ DC } & \multirow{2}{*}{$\begin{array}{c}\text { Parameters remain } \\
\text { within post-irradiation } \\
\text { specification. } \\
\text { Completed 11/22/2016. }\end{array}$} & \multirow[b]{2}{*}{$\mathrm{Y}$} & 1 & $>40$ \\
\hline & & & & & & & & 0.5 & $>30$ \\
\hline \multirow{3}{*}{ RHF43B } & \multirow{3}{*}{ STMicroelectronics } & \multirow{3}{*}{ 30820A; (A589) } & \multirow{3}{*}{$\begin{array}{c}\text { Operational } \\
\text { Amplifier }\end{array}$} & \multirow{3}{*}{$\begin{array}{l}\text { Bipolar / } \\
\text { Ceramic } \\
\text { Flat-8 }\end{array}$} & & Minimal dose rate & & 10 & $>100$ \\
\hline & & & & & DC & sensitivity. Parameters & $\mathrm{N}$ & 1 & $>50$ \\
\hline & & & & & & $12 / 16 / 16$ & & 0.5 & $>50$ \\
\hline
\end{tabular}




\begin{tabular}{|c|c|c|c|c|c|c|c|c|c|}
\hline Part Number & Manufacturer & LDC & $\begin{array}{l}\text { Device } \\
\text { Function }\end{array}$ & $\begin{array}{l}\text { Technology } \\
\text { /Package }\end{array}$ & PI & Results & $\begin{array}{l}\text { App. } \\
\text { Spec } \\
(\mathbf{Y} / \mathbf{N})\end{array}$ & $\begin{array}{c}\text { Dose rate } \\
(\operatorname{mrad}(\mathrm{Si}) / \mathrm{s})\end{array}$ & $\begin{array}{l}\text { Degradation } \\
\text { Level (krad } \\
\text { (Si)) }\end{array}$ \\
\hline \multicolumn{10}{|l|}{ TRANSISTORS } \\
\hline \multirow{3}{*}{ 2N2222 } & \multirow{3}{*}{ Semicoa } & \multirow{3}{*}{ 1001; (13-024) } & \multirow{3}{*}{$\begin{array}{c}\text { NPN } \\
\text { Transistor }\end{array}$} & \multirow{3}{*}{$\begin{array}{l}\text { Bipolar / } \\
\text { Engineering } \\
\text { Samples }\end{array}$} & \multirow{3}{*}{ DC } & \multirow{3}{*}{$\begin{array}{l}\text { Minimal degradation. } \\
\text { All parameters within } \\
\text { spec. [43] }\end{array}$} & \multirow{3}{*}{$\mathrm{N}$} & 10 & $>100$ \\
\hline & & & & & & & & 1 & $>40$ \\
\hline & & & & & & & & 0.5 & $>20$ \\
\hline \multirow{4}{*}{ 2N2222AJSR } & \multirow{4}{*}{ Semicoa } & \multirow{4}{*}{ 1364; (13-017) } & \multirow{4}{*}{$\begin{array}{c}\text { NPN } \\
\text { Transistor }\end{array}$} & \multirow{4}{*}{ Bipolar } & \multirow{4}{*}{ DC } & \multirow{4}{*}{$\begin{array}{l}\text { LDR EF = } 3.9 \text { After } \\
100 \text { krad(Si). } \\
\text { Completed in } 2016 .\end{array}$} & \multirow{4}{*}{$\mathrm{N}$} & 10 & $35<\mathrm{h}_{\mathrm{FE}}<45$ \\
\hline & & & & & & & & 5 & $65<\mathrm{h}_{\mathrm{FE}}<90$ \\
\hline & & & & & & & & 1 & $>40$ \\
\hline & & & & & & & & 0.5 & $>30$ \\
\hline \multirow[b]{2}{*}{ 2N3811JS } & \multirow[b]{2}{*}{ Semicoa } & \multirow[b]{2}{*}{ 1230; (13-063) } & \multirow[b]{2}{*}{$\begin{array}{c}\text { PNP } \\
\text { Transistor }\end{array}$} & \multirow[b]{2}{*}{ Bipolar } & \multirow[b]{2}{*}{ DC } & No bias dependence. & \multirow[b]{2}{*}{$\mathrm{N}$} & 1 & $30<\mathrm{h}_{\mathrm{FE}}<50$ \\
\hline & & & & & & $\begin{array}{c}\text { Two devices exceeded } \\
\text { specifications after } 30 \\
\text { krad(Si). Completed } \\
12 / 3 / 2016 .\end{array}$ & & 0.5 & $60<\mathrm{h}_{\mathrm{FE}} \leq 70$ \\
\hline 2N2907 & Semicoa & 0932; (13-023) & $\begin{array}{c}\text { PNP } \\
\text { Transistor }\end{array}$ & Bipolar & DC & $\begin{array}{c}\text { LDR EF = } 1.78 \text { after } \\
100 \text { krad(Si). } \\
\text { Completed 12/3/2016. }\end{array}$ & $\mathrm{N}$ & 10 & $40<\mathrm{h}_{\mathrm{FE}}<50$ \\
\hline 2N2369 & Semicoa & $\begin{array}{c}\text { J1934(wafer\#); (13- } \\
\text { 020) }\end{array}$ & $\begin{array}{c}\text { NPN } \\
\text { Transistor }\end{array}$ & Bipolar & DC & $\begin{array}{l}\text { All parameters within } \\
\text { specification up to } 100 \\
\text { krad(Si). Minimal LDR } \\
\text { sensitivity. Completed } \\
\text { Nov. } 2016 \\
\end{array}$ & $\mathrm{~N}$ & 1 & $>100$ \\
\hline \multirow[b]{2}{*}{ 2N3700JV } & \multirow[b]{2}{*}{ Semicoa } & \multirow[b]{2}{*}{ 1109; (13-022) } & \multirow[b]{2}{*}{$\begin{array}{c}\text { NPN } \\
\text { Transistor }\end{array}$} & \multirow[b]{2}{*}{ Bipolar } & \multirow[b]{2}{*}{ DC } & \multirow{2}{*}{$\begin{array}{c}\text { Strong bias } \\
\text { dependence. Biased } \\
\text { devices show enhanced } \\
\text { degradation than } \\
\text { grounded devices. } \\
\text { Completed 6/23/2016. }\end{array}$} & \multirow[b]{2}{*}{$\mathrm{N}$} & 1 & $30<\mathrm{h}_{\mathrm{FE}}<40$ \\
\hline & & & & & & & & 0.5 & $>20$ \\
\hline \multirow{2}{*}{ 2N3700UBJV } & \multirow{2}{*}{ Semicoa } & \multirow{2}{*}{$\begin{array}{l}\mathrm{J} 1935 \text { (wafer\#); (13- } \\
\text { 021) }\end{array}$} & NPN & D. & & Dose rate effect not & & 1 & $10<\mathrm{h}_{\mathrm{FE}}<20$ \\
\hline & & & Transistor & Bipolar & DC & $\begin{array}{l}\text { evident at this stage. } \\
\text { Completed 6/23/2016. }\end{array}$ & $\mathrm{N}$ & 0.5 & $15<\mathrm{h}_{\mathrm{FE}}<30$ \\
\hline 2N5153 & Semicoa & 1013; (13-018) & $\begin{array}{c}\text { PNP } \\
\text { Transistor }\end{array}$ & Bipolar & DC & $\begin{array}{c}\text { Minimal LDR EF. } \\
\text { Completed 11/22/2016. }\end{array}$ & $\mathrm{N}$ & 1 & $>50$ \\
\hline 2N5154 & Semicoa & 1023; (13-019) & $\begin{array}{c}\text { NPN } \\
\text { Transistor }\end{array}$ & Bipolar & DC & $\begin{array}{c}\text { Minimal LDR EF. } \\
\text { Completed 11/22/2016. }\end{array}$ & $\mathrm{N}$ & 1 & $>50$ \\
\hline
\end{tabular}




\begin{tabular}{|c|c|c|c|c|c|c|c|c|c|}
\hline Part Number & Manufacturer & LDC & $\begin{array}{c}\text { Device } \\
\text { Function }\end{array}$ & $\begin{array}{l}\text { Technology } \\
\text { /Package }\end{array}$ & PI & Results & $\begin{array}{l}\text { App. } \\
\text { Spec } \\
\text { (Y/N) }\end{array}$ & $\begin{array}{c}\text { Dose rate } \\
(\operatorname{mrad}(\mathrm{Si}) / \mathrm{s})\end{array}$ & $\begin{array}{c}\text { Degradation } \\
\text { Level (krad } \\
\text { (Si)) }\end{array}$ \\
\hline \multicolumn{10}{|c|}{ VOLTAGE REFERENCES/REGULATORS } \\
\hline LM136AH2.5QMLV & $\begin{array}{c}\text { National } \\
\text { Semiconductor }\end{array}$ & $\begin{array}{l}\text { 200746K019; } \\
\text { (A164) }\end{array}$ & $\begin{array}{l}\text { Voltage } \\
\text { Reference }\end{array}$ & $\begin{array}{l}\text { Bipolar/3- } \\
\text { LEAD TO- } \\
\quad 46\end{array}$ & $\mathrm{DC}$ & $\begin{array}{l}\text { Exhibits no LDR } \\
\text { enhancement. }\end{array}$ & $\mathrm{N}$ & 0.5 & $>70$ \\
\hline LM317LTTR & Texas Instruments & 0608; (A113) & $\begin{array}{l}\text { Positive } \\
\text { Voltage } \\
\text { Regulator }\end{array}$ & Bipolar & DC & $\begin{array}{l}\text { Parameters within } \\
\text { specification. Observed } \\
\text { LDR sensitivity for } \\
\text { parts irradiated at } 0.5 \\
\text { after } 20 \text { krad(Si). }\end{array}$ & $\mathrm{N}$ & 0.5 & $>70$ \\
\hline LT1009IDR & Texas Instruments & 0606; (A327) & $\begin{array}{l}\text { Internal } \\
\text { Reference }\end{array}$ & Bipolar & DC & $\begin{array}{l}\text { Parameters within } \\
\text { specification. Parts } \\
\text { exhibit minimal LDR } \\
\text { enhancement. }\end{array}$ & $\mathrm{N}$ & 0.5 & $>70$ \\
\hline RHFL4913ESY332 & STMicroelectronics & 30828A; (A259) & $\begin{array}{l}\text { Voltage } \\
\text { Regulator }\end{array}$ & $\begin{array}{c}\text { Bipolar/TO- } \\
257\end{array}$ & DC & $\begin{array}{l}\text { All parameters within } \\
\text { specification. Minimal } \\
\text { dose rate sensitivity. } \\
\text { Completed } 7 / 22 / 2016 \text {. }\end{array}$ & $\mathrm{N}$ & 0.5 & $>60$ \\
\hline RHFL4913KP332 & STMicroelectronics & 30814B; (A258) & $\begin{array}{l}\text { Voltage } \\
\text { Regulator }\end{array}$ & $\begin{array}{c}\text { Bipolar/Flat- } \\
16\end{array}$ & DC & $\begin{array}{l}\text { All parameters within } \\
\text { specification. Minimal } \\
\text { dose rate sensitivity. } \\
\text { Completed } 7 / 22 / 2016 \text {. }\end{array}$ & $\mathrm{N}$ & 0.5 & $>60$ \\
\hline TL750M05CKTRR & Texas Instruments & 0707; (A112) & $\begin{array}{c}\text { LDO } \\
\text { Positive } \\
\text { Voltage } \\
\text { Regulator }\end{array}$ & $\begin{array}{c}\text { Bipolar/TO- } \\
263-3\end{array}$ & DC & $\begin{array}{l}\text { Minimal dose rate } \\
\text { sensitivity. }\end{array}$ & $\mathrm{N}$ & 0.5 & $>70$ \\
\hline \multicolumn{10}{|l|}{ MISCELLANEOUS } \\
\hline LM139AWRQMLV & $\begin{array}{c}\text { National } \\
\text { Semiconductor }\end{array}$ & JM046X13; (A211) & Comparator & Bipolar & DC & $\begin{array}{c}\text { Parameters within } \\
\text { specification. } \\
\text { Completed 11/22/2016. }\end{array}$ & $\mathrm{Y}$ & 0.5 & $\mathrm{Ib}>75$ \\
\hline
\end{tabular}


TABLE V: SummaRy of DD TEST RESUltS

\begin{tabular}{|c|c|c|c|c|c|c|c|c|}
\hline Part Number & Manufacturer & LDC & $\begin{array}{l}\text { Device } \\
\text { Function }\end{array}$ & Technology & PI & Results & $\begin{array}{c}\text { App. } \\
\text { Spec } \\
(\mathbf{Y} / \mathbf{N})\end{array}$ & Proton Fluence $\left(/ \mathrm{cm}^{2}\right)$ \\
\hline SW15-802 & $\begin{array}{l}\text { Southwest Research } \\
\text { Institute }\end{array}$ & $\begin{array}{l}1203,1233 ; \\
(16-007)\end{array}$ & Optocoupler & Hybrid & MCC & $\begin{array}{l}\text { Increase of dark current and decrease } \\
\text { of CTR with increasing fluence }\end{array}$ & Y & $6 \times 10^{10}<\mathrm{I}_{\text {off }}<3 \times 10^{11}$ \\
\hline HSSR-7111 & Micropac & $1614 ;(16-035)$ & Optocoupler & Hybrid & MJC & $\begin{array}{l}\text { Some degradation in turn on time, } \\
\text { leakage prevents turn off }\end{array}$ & Y & $3 \times 10^{11}<\mathrm{I}_{\text {off }}<4 \times 10^{11}$ \\
\hline OРB848 & Optek & n/a; (17-009) & Optocoupler & Hybrid & MJC & $\begin{array}{l}\text { On-state collector current out of } \\
\text { specification at } \\
9.32 \times 10^{10}<\mathrm{I}_{\text {Con }}<1.12 \times 10^{11}\end{array}$ & $\mathrm{Y}$ & $9.32 \times 10^{10}<\mathrm{I}_{\text {Con }}<1.12 \times 10^{11}$ \\
\hline
\end{tabular}




\section{TEST RESULTS AND DISCUSSION}

As in our past workshop compendia of GSFC test results, each device under test has a detailed test report available online at http://radhome.gsfc.nasa.gov [3] and at http://nepp.nasa.gov [4] describing in further detail the test method, conditions and monitored parameters, and test results. This section contains a summary of testing performed on a selection of featured parts.

\section{A. AD9364, Analog Devices, RF Transceiver}

The AD9364 is a commercial-off-the-shelf (COTS) high performance, highly integrated radio frequency (RF) Agile Transceiver designed for use in $3 \mathrm{G}$ and $4 \mathrm{G}$ base station applications. It is built on a commercial 65-nm CMOS process. TID testing was carried out on four samples at an average dose rate of $100 \mathrm{rad}(\mathrm{Si}) / \mathrm{s}$.

The device under test (DUT) was configured as a part of the AD-FMCOMMS4-EBZ evaluation platform. The ADFMCOMMS4-EBZ evaluation platform interfaced with the ZedBoard. The ZedBoard contains the Zynq-7020 Systemon-Chip (SoC), 512 MB DDR3, 256 Mb Quad-SPI flash, and 4 GB SD memory card. Figure 1 shows a photograph of the test setup on the bench, with the evaluation cards mated with the ZedBoard. Figure 2 shows the top and bottom view of the evaluation board. The AD9364 is circled. As shown, there are several other active components mounted on the bottom of the board. During irradiation, the entire ZedBoard was positioned behind lead brick shielding. We performed dosimetry at various locations behind the shielding, and determined that at a spot one inch away from the edge of the shielding, the total dose is negligible. However, approximately $1 / 2$ inch away from the edge, the total dose will be a fifth of the total dose received by the DUT at the unshielded target. Therefore, the components on the evaluation card near the edge of the shielding accumulated approximately $5-10 \operatorname{krad}(\mathrm{Si})$ during the exposure.

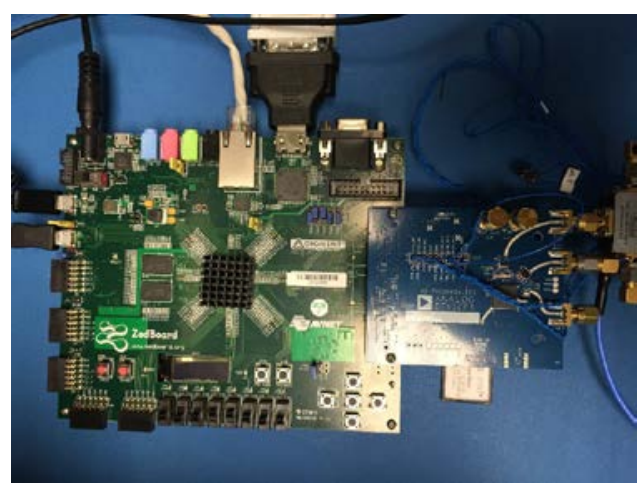

Fig. 1. Photograph of the test setup.

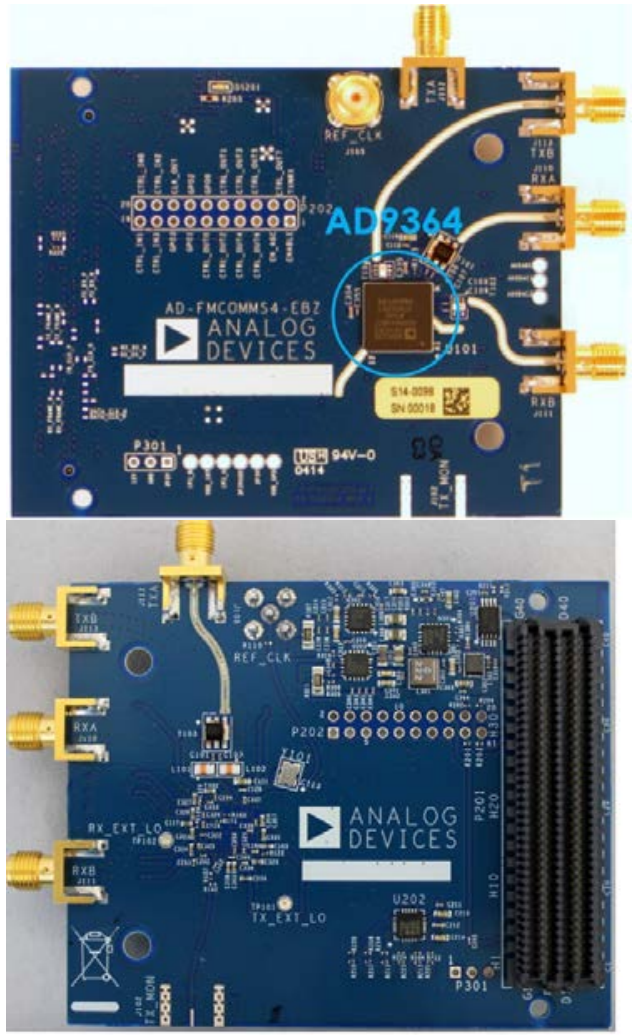

Fig. 2. Top and bottom view of the AD-FMCOMMS4EBZ evaluation board.

The parts exhibited limited degradation in general. Most of the electrical parameters showed negligible change up to $50 \mathrm{krad}(\mathrm{Si})$. The transmission power gain showed some degradation with increasing total dose. The gain degradation manifested visually through the image transmission tests. Figure 3 shows a pristine image and an image transmitted with a gain of $62 \mathrm{~dB}$ after $50 \mathrm{krad}(\mathrm{Si})$. We show the results from two transmission operations. The second test, shown in Figure 3b, produced relatively fewer errors. In both cases, the transmitted image postirradiation becomes pixelated due to the loss in power. Figure 3, third image, shows the pre-irradiation and postirradiation image with a gain of $50 \mathrm{~dB}$. The pixelation is reduced significantly. The pixelation issue disappears at a higher transmission power. 


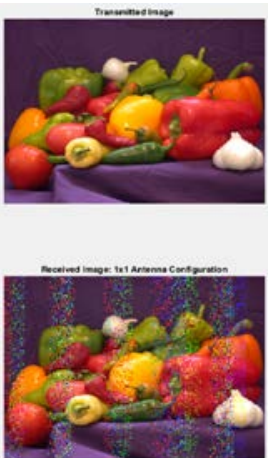

a. First
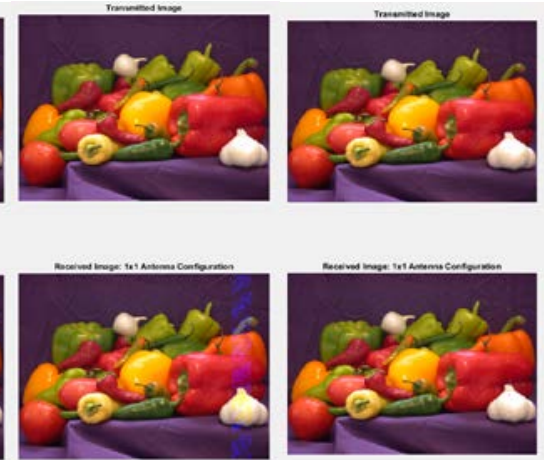

b. Second

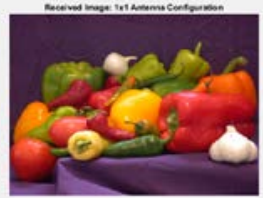

c. Third
Fig. 3. Pre-irradiation (top) and post-irradiation (bottom) images transmitted after $50 \mathrm{krad}(\mathrm{Si})$ for DUT2. The first and second images represent the first (a) and second (b) transmissions, respectively. The second transmission produced relatively fewer errors. The third image transmitted (c) with gain of $50 \mathrm{~dB}$ after $50 \mathrm{krad}(\mathrm{Si})$ for DUT2.

\section{B. HSSR-7111, Micropac, Optocoupler}

The HSSR-7111 is a single-channel power MOSFET optocoupler rated for $90 \mathrm{~V}$. It is available to Standard Microcircuit Drawings (SMD) specifications as 59629314001HPA. Displacement Damage testing was conducted on ten samples at CNL-UCD. To avoid part overstress a Keithley Pulse Measurement Unit (PMU) was used for pulse sweeping the device parameters, this also reduces internal heating of the device. During testing we saw two types of degradation on the optocoupler, increased turn on time delay and leakage on the output MOSFET.

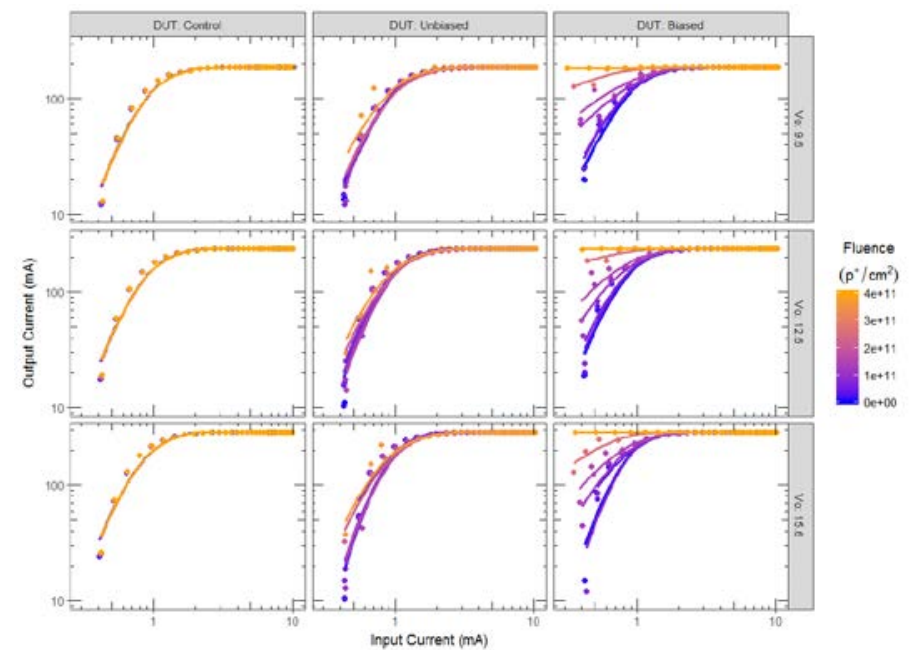

Fig. 4. Output current for a given input current using pulsed measurements.

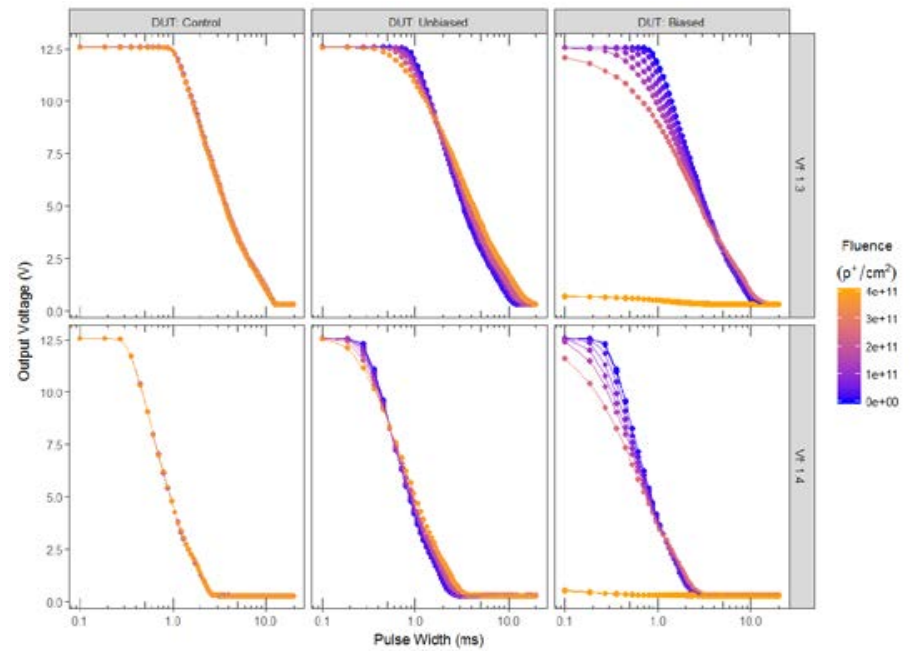

Fig. 5. Turn-on voltage for a given pulse width.

The degradation shown in Figure 4 details the biased parts as being more susceptible to proton exposure. At the final tested fluence step, the devices were permanently "on" independent of input current. This effect is attributed to the leakage path through the Metal Oxide Semiconductor Field Effect Transistor (MOSFET) stage of the device. This degradation remained present even as no bias was on the LED stage. Figure 5 however shows the delay in turn on time for given pulse widths synchronized on the drain of the MOSFET and high side of the LED. The delay is more pronounced for the unbiased devices, and therefore is suspected to be degradation of the LED and/or material that the light propagates through.

\section{SW15-802, Southwest Research Institute, High Voltage Optocoupler}

The SW15-802 is a $\pm 6 \mathrm{kV}$ optocoupler with heterojunction LED structure. It employs low outgassing spacegrade potting and coating. The internal high-voltage diode is glass-passivated and rated at $15 \mathrm{kV}$. Figure 6 shows a pin configuration for this uniquely packaged device. Southwest Research Institute designed this optocoupler as a replacement for a commercially-available radiationtolerant optocoupler.

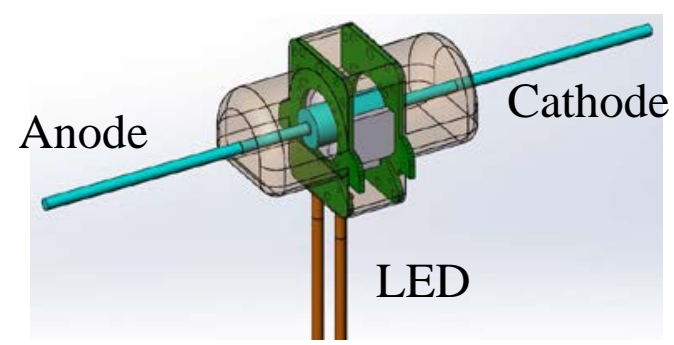

Fig. 6. Pin configuration and description.

TID testing was carried out on eight parts to a total dose of $150 \mathrm{krad}(\mathrm{Si})$. The four unbiased parts, with all pins grounded, were irradiated first at a dose rate of 50 $\operatorname{rad}(\mathrm{Si}) / \mathrm{s}$. Only small degradation was observed up to 75 $\operatorname{krad}(\mathrm{Si})$. After this dose step, the dark current parameter 
for one part increased ten times from the pre-irradiation value. These results are shown in Figure 7. Only small increases in the CTR measurement were observed for LED current conditions of $10 \mathrm{~mA}$ and $20 \mathrm{~mA}$. A large CTR increase was seen with an LED current of $0 \mathrm{~mA}$, almost ten times the pre-radiation value.

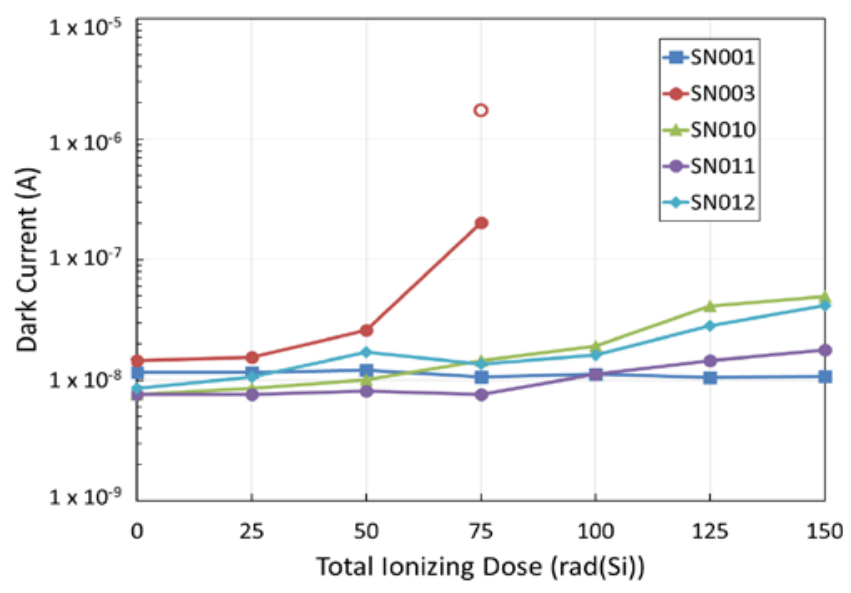

Fig. 7. Dark current versus TID for the parts irradiated unbiased.

The remaining four parts were biased at $\pm 6.1 \mathrm{kV}$ on the anode and cathode and no LED bias current. All four parts were irradiated together up to $7.6 \mathrm{krad}(\mathrm{Si})$. At this point, the parts were drawing too much current for the $10 \mathrm{kV}$ Stanford Research Systems power supply. One part was then irradiated at a time and the dose rate was reduced from $50 \mathrm{rad}(\mathrm{Si}) / \mathrm{s}$ to about $5 \mathrm{rad}(\mathrm{Si}) / \mathrm{s}$. SN056 showed almost a thirty times increase in the dark current parameter at $7.6 \mathrm{krad}(\mathrm{Si})$. The open circle shown for SN056 are measurements of the part that were taken four days later after a 60C annealing. There was some recovery, but the dark current still shows about a twenty times increase compared to the pre-rad value. This confirmed that the parts did experience some recovery from annealing, but most of the damage remained. The other three irradiated parts each had about 10x increase in dark current. Figure 8 shows the biased parts results for the dark current parameter. Similar results were seen in the biased parts for CTR as in the unbiased parts. At an LED current of $0 \mathrm{~mA}$ the CTR increased by thirty-eight times, while the LED current conditions of $10 \mathrm{~mA}$ and $20 \mathrm{~mA}$ only saw about a $5 \%$ increase.

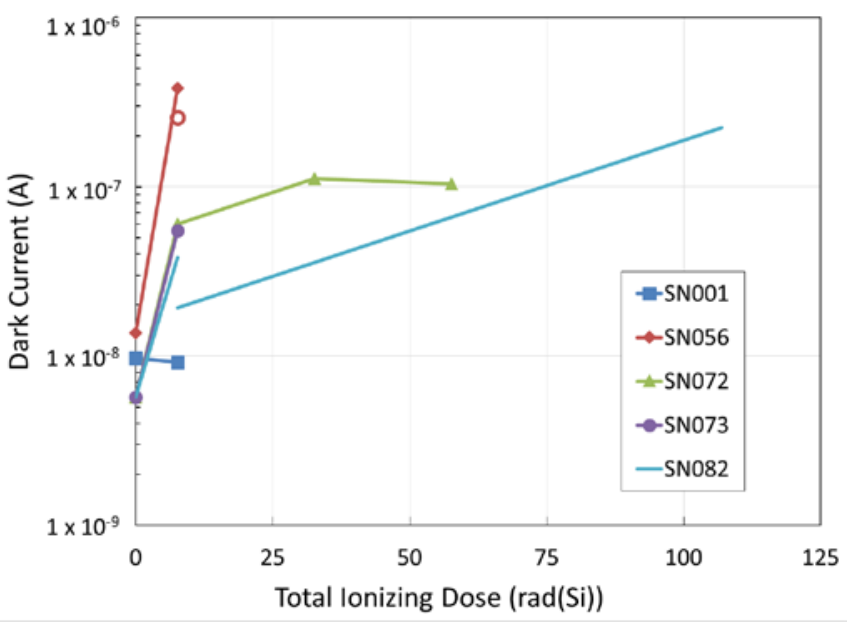

Fig. 8. Dark current versus TID for the parts irradiated while biased at $\pm 6.1 \mathrm{kV}$.

Displacement damage testing was also conducted on eight parts at TAMU. Four parts were irradiated with diode and LED grounded and the remaining four parts were irradiated with the diode grounded but the LED biased at $20 \mathrm{~mA}$. There were two control devices. The parts were irradiated in $6 \times 10^{10} \mathrm{p} / \mathrm{cm}^{2}$ steps up to a total fluence of $3 \times 10^{11} \mathrm{p} / \mathrm{cm}^{2}$.

In both the biased and unbiased parts, dark current increased as the proton fluence increased. Figures 9 and 10 show these test results. Similar results were also seen in the CTR parameter. CTR decreased as fluence increased when the LED current conditions were $10 \mathrm{~mA}$ and $20 \mathrm{~mA}$ but increased when the LED current was $0 \mathrm{~mA}$. Only failure of this optocoupler was an unacceptable response as there was no defined specification limit.

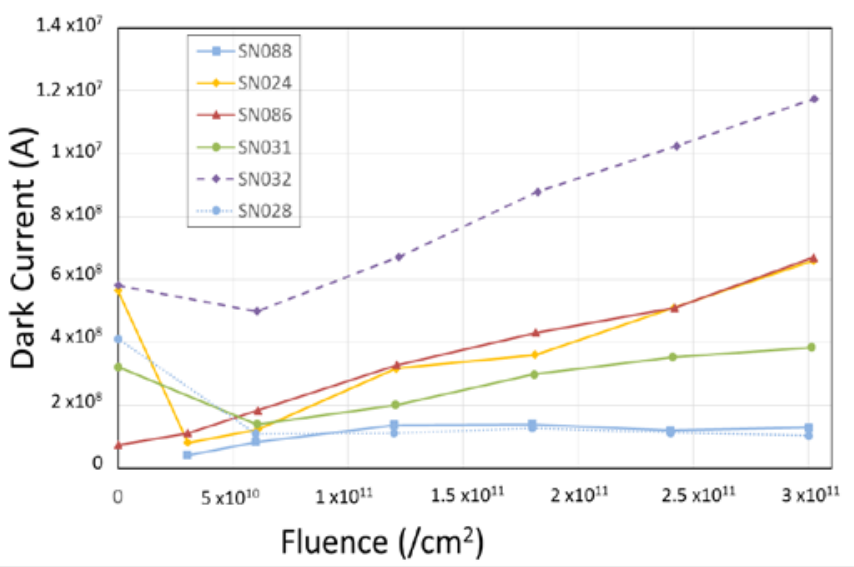

Fig. 9. Dark current as a function of proton fluence for the unbiased parts. 


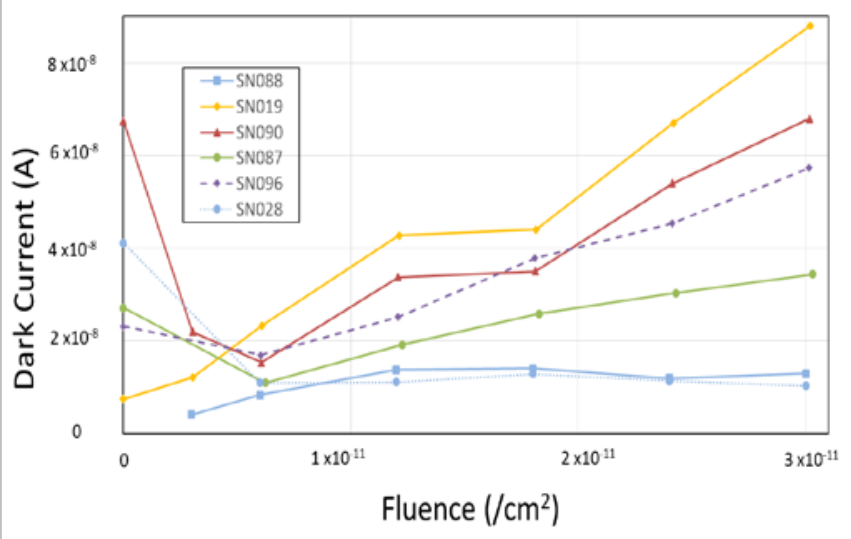

Fig. 10. Dark current as a function of proton fluence for the biased parts.

\section{SUMMARY}

We have presented data from recent TID tests on a variety of primarily commercial devices. It is the authors' recommendation that this data be used with caution due to many application/lot-specific issues. We also highly recommend that lot testing be performed on any suspect or commercial device. As in our past workshop compendia of GSFC test results, each DUT has a detailed test report available online describing in further detail, test method, TID conditions/parameters, test results, and graphs of data [3].

\section{ACKNOWLEDGMENT}

The authors acknowledge the sponsors of this effort: NASA Electronic Parts and Packaging Program (NEPP), and NASA Flight Projects. The authors thank members of the Radiation Effects and Analysis Group (REAG) who contributed to the test results presented here: Melanie D. Berg, Stephen K. Brown, Martin A. Carts, Stephen R. Cox, James D. Forney, Yevgeniy Gerashchenko, Hak S. Kim, Anthony M. Phan, and Christina M. Seidleck.

The authors would also like to thank all external assistance in testing the high voltage optocoupler (SW15802). We acknowledge Eric Young and Emanuel Hernandez of the Power group at NASA GSFC, code 563 and Carlos Urdiales, Armando De Los Santos, Dennis Guerrero, Steve Persyn, Mark Phillips, and Jessica Tumlinson, of Southwest Research Institute. Without their expertise and support, none of this work would have been possible.

\section{REFERENCES}

[1] Martha V. O’Bryan, Kenneth A. LaBel, Carl M. Szabo, Dakai Chen, Michael J. Campola, Megan C. Casey, Jean-Marie Lauenstein, Edward J. Wyrwas, Steven M. Guertin, Jonathan A. Pellish, and Melanie D. Berg, "Compendium of Current Single Event Effects Results from NASA Goddard Space Flight Center and NASA Electronic Parts and Packaging Program," submitted for publication in IEEE Radiation Effects Data Workshop, Jul. 2017.

[2] Department of Defense "Test Method Standard Microcircuits," MIL-STD-883 Test Method 1019.9 Ionizing radiation (total dose) test procedure, June 7, 2013, https://landandmaritimeapps.dla.mil/Downloads/MilSpec/Docs/MIL -STD-883/std883.pdf.

[3] NASA/GSFC Radiation Effects and Analysis home page, http://radhome.gsfc.nasa.gov.

[4] NASA Electronic Parts and Packaging Program home page, http://nepp.nasa.gov. 